\title{
Some translation-invariant Banach function spaces which contain $c_{0}$
}

\author{
by \\ P. Lefèvre (Lens), D. Li (Lens), H. Queffélec (Lille) \\ and L. Rodríguez-Piazza (Sevilla)
}

\begin{abstract}
We produce several situations where some natural subspaces of classical Banach spaces of functions over a compact abelian group contain the space $c_{0}$.

I. Introduction. Let $G$ be a compact abelian group and $\Gamma=\widehat{G}$ its dual group. It is a familiar theme in Harmonic Analysis to compare the "thinness" properties of a subset $\Lambda \subseteq \Gamma$ with the Banach space properties of the space $X_{\Lambda}$, where $X$ is a Banach space of Haar-integrable functions on $G$ and $X_{\Lambda}$ is the subspace of $X$ consisting of the $f \in X$ whose spectrum lies in $\Lambda$ : $\widehat{f}(\gamma)=0$ if $\gamma \notin \Lambda$. We refer to Kwapień-Pełczyński's classical paper [17] for such investigations.

It is known that, with $\Psi_{2}$ denoting the Orlicz function $e^{x^{2}}-1$ :

(1) If $L_{\Lambda}^{\Psi_{2}}=L_{\Lambda}^{2}$, then $\Lambda$ is a Sidon set (Pisier [35, Théorème 6.2]).

(2) If $\mathcal{C}_{\Lambda}$ has a finite cotype, then $\Lambda$ is a Sidon set (Bourgain-Milman [3]).
\end{abstract}

Recall that $\Lambda$ is a Sidon set if every continuous function on $G$ with spectrum in $\Lambda$ has an absolutely convergent Fourier series.

In a previous paper, we proved, among other facts, the following extension of (1) ([19, Theorem 2.3]):

$\left(1^{\prime}\right)$ If $L_{\Lambda}^{\Psi_{2}}$ has cotype 2 , then $\Lambda$ is a Sidon set.

We also showed the following variant of (2) ([19, Theorem 1.2]):

$\left(2^{\prime}\right)$ If $U_{\Lambda}$ has a finite cotype, then $\Lambda$ is a Sidon set.

Here $U=U(\mathbb{T})$ is the space of continuous functions on the circle group $\mathbb{T}$ whose Fourier series converges uniformly on $\mathbb{T}$.

2000 Mathematics Subject Classification: Primary 43A46, 46B20; Secondary 42A55, 42B35, 43A07, 46E30. 
In this work, we study the implications on $\Lambda$ of the fact that some Banach space $X_{\Lambda}$ contains, or not, the space $c_{0}$. In particular, we extend $\left(1^{\prime}\right)$ and $\left(2^{\prime}\right)$.

The paper is organized as follows. In Section II, we show that if $\psi$ is an Orlicz function which violates the $\Delta_{2}$-condition, in a strong sense: $\lim _{x \rightarrow+\infty} \psi(2 x) / \psi(x)=+\infty$ (which is the case for $\Psi_{2}$ ), and if $X_{0}$ is a linear subspace of $L^{\infty}$ on which the norms \|\|$_{2}$ and \|\|$_{\psi}$ are not equivalent, then the closure $X$ of $X_{0}$ in $L^{\psi}$ contains $c_{0}$. It follows that if $\Lambda$ is not a Sidon set, then $L_{\Lambda}^{\Psi_{2}}$ contains $c_{0}$, and a fortiori that if $L_{\Lambda}^{\Psi_{2}}$ has a finite cotype, then $\Lambda$ is a Sidon set, which generalizes $\left(1^{\prime}\right)$.

In Section III, we extend $\left(2^{\prime}\right)$ by showing that: If $\Lambda$ is not a set of uniform convergence (i.e. if $U_{\Lambda} \neq \mathcal{C}_{\Lambda}$ ), then $U_{\Lambda}$ does contain $c_{0}$. In particular, if $U_{\Lambda}$ has a finite cotype, then $U_{\Lambda}=\mathcal{C}_{\Lambda}$, so $\mathcal{C}_{\Lambda}$ has a finite cotype and therefore, in view of (2), $\Lambda$ is a Sidon set. This explains why the proof of $\left(2^{\prime}\right)$ in [19] mimicked Bourgain and Milman's.

In Section IV, we use the notion of invariant mean in $L^{\infty}(G)$. We say that $\Lambda$ is a Lust-Piquard set if, for every function $f \in L_{\Lambda}^{\infty}$, the product $\gamma f$ of $f$ with every character $\gamma \in \Gamma$ has a unique invariant mean. Of course, if every $f \in L_{\Lambda}^{\infty}$ is continuous (i.e. $\Lambda$ is a Rosenthal set), then $\Lambda$ is a Lust-Piquard set. F. Lust-Piquard ([27]) showed that there are Lust-Piquard sets which are not Rosenthal sets, and, more precisely, that $\Lambda=\mathbb{P} \cap(5 \mathbb{Z}+2)$, where $\mathbb{P}$ is the set of the prime numbers, is a Lust-Piquard set such that $\mathcal{C}_{\Lambda}$ contains $c_{0}$ (if $\Lambda$ is a Rosenthal set, $\mathcal{C}_{\Lambda}$ cannot contain $c_{0}$ ). We construct here another kind of "big" Lust-Piquard set $\Lambda$, namely a Hilbert set. Then $\mathcal{C}_{\Lambda}$ contains $c_{0}$ by a result of the second-named author ([22, Theorem 2]).

In Section $V$, we investigate conditions under which the space $\mathcal{C}_{\Lambda}$ is complemented in $L_{\Lambda}^{\infty}$. We conjecture that this happens only if $\mathcal{C}_{\Lambda}=L_{\Lambda}^{\infty}$, i.e. $\Lambda$ is a Rosenthal set. We are only able to show that, under that condition of complementation, $\mathcal{C}_{\Lambda}$ does not contain $c_{0}$, and, moreover, every $f \in L_{\Lambda}^{\infty}$ which is Riemann-integrable is actually in $\mathcal{C}_{\Lambda}$.

Notation. Throughout this paper, $G$ is a compact abelian group, and $\Gamma=\widehat{G}$ is its (discrete) dual group. The Haar measure of $G$ is denoted by $m$, and integration with respect to $m$ by $d t$ or $d x$. We shall write the group structure of $\Gamma$ additively, so that, for $\gamma \in \Gamma$, the character $-\gamma \in \Gamma$ is the function $\bar{\gamma} \in \mathcal{C}(G)$. When $G$ is the circle group $\mathbb{T}=\mathbb{R} / 2 \pi \mathbb{Z}$, we identify, as usual, the character $e_{n}: t \mapsto e^{\text {int }}$ with the integer $n \in \mathbb{Z}$, and so the dual group $\Gamma$ with $\mathbb{Z}$; the Haar measure is then $d t / 2 \pi$.

For $f \in L^{1}(G)$, the Fourier coefficient of $f$ at $\gamma \in \Gamma$ is $\widehat{f}(\gamma)=$ $\int_{G} f(t) \overline{\gamma(t)} d t$. If $X$ is a linear function subspace of $L^{1}(G)$, we denote by $X_{\Lambda}$ the subspace of those $f \in X$ for which the Fourier coefficients vanish outside of $\Lambda$. 
When we say that a Banach space $X$ contains a Banach space $Y$, we mean that $X$ contains a (closed) subspace isomorphic to $Y$.

Acknowledgements. This work was partly supported by a Picasso project (EGIDE-MCYT) between the French and Spanish governments.

We thank the referee for a very careful reading of this paper and for many suggestions to improve the writing.

II. Subspaces of Orlicz spaces. Let $\psi$ be an Orlicz function, that is, an increasing convex function $\psi:[0,+\infty[\rightarrow[0,+\infty[$ such that $\psi(0)=0$ and $\psi(+\infty)=+\infty$. We shall assume that $\psi$ violates the $\Delta_{2}$-condition, in the following strong sense:

$$
\lim _{x \rightarrow+\infty} \frac{\psi(2 x)}{\psi(x)}=+\infty .
$$

Let $(\Omega, \mathcal{A}, \mathbb{P})$ be a probability space. The Orlicz space $L^{\psi}(\Omega)$ is the space of all (equivalence classes of) measurable functions $f: \Omega \rightarrow \mathbb{C}$ for which there is a constant $C \geq 0$ such that

$$
\int_{\Omega} \psi\left(\frac{|f(t)|}{C}\right) d \mathbb{P}(t) \leq 1
$$

and then $\|f\|_{\psi}$ is the least possible constant $C$.

Observe that $(*)$ implies that there exists $a>0$ such that $\psi(2 t) \geq 4 \psi(t)$ for every $t \geq a$. Hence, for all $n \geq 0$, one has $\psi\left(2^{n} a\right) \geq 4^{n} \psi(a)$. It follows that for $2^{n} a \leq x<2^{n+1} a$, we have

$$
\psi(x) \geq \psi\left(2^{n} a\right) \geq 4^{n} \psi(a) \geq\left(\frac{x}{2 a}\right)^{2} \psi(a)=C x^{2} .
$$

Hence $\psi(x) \geq C x^{2}$ for every $x \geq a$, and so the norm \|\|$_{\psi}$ is stronger than the norm of $L^{2}$.

TheOREM II.1. Suppose that $\psi$ is an Orlicz function as above. Let $X_{0}$ be a linear subspace of $L^{\infty}(\Omega)$ on which the norms \|\|$_{2}$ and \|\|$_{\psi}$ are not equivalent. Then there exists in $X_{0}$ a sequence which is equivalent, in the closure $X$ of $X_{0}$ for the norm \|\|$_{\psi}$, to the canonical basis of $c_{0}$.

Proof. We first remark that, thanks to $(*)$, we can choose, for each $n \geq 1$, a positive number $x_{n}$ such that

$$
\psi\left(\frac{x}{2}\right) \leq \frac{1}{2^{n}} \psi(x), \quad \forall x \geq x_{n} .
$$

Since $\psi$ increases, for every $x \geq 0$ we have

$$
\psi\left(\frac{x}{2}\right) \leq \frac{1}{2^{n}} \psi(x)+\psi\left(x_{n}\right) .
$$


Next, $\psi$ is continuous since it is convex. Hence there exists $a>0$ such that $\psi(a)=1$. Then, since $\psi$ is increasing, for every $f \in L^{\infty}(\Omega)$ we have

$$
\int_{\Omega} \psi\left(a \frac{|f|}{\|f\|_{\infty}}\right) d \mathbb{P} \leq 1
$$

and so $\|f\|_{\psi} \leq(1 / a)\|f\|_{\infty}$.

Now, let $\alpha_{n}, n \geq 1$, be positive numbers less than $a / 2$ such that $\sum_{n \geq 1} \alpha_{n}$ $<a$. We shall construct inductively a sequence of functions $f_{n} \in X_{0}$, with $\left\|f_{n}\right\|_{\psi}=1$, and a sequence of positive numbers $\beta_{n} \leq 1 / 2^{n}$ such that:

(i) $\mathbb{P}\left(\left\{\left|f_{n}\right|>\alpha_{n}\right\}\right) \leq \beta_{n}$ for every $n \geq 1$;

(ii) if we set $M_{1}=1$ and

$$
M_{n}=\psi\left(\frac{\left\|f_{1}\right\|_{\infty}+\cdots+\left\|f_{n-1}\right\|_{\infty}}{2}\right) \quad \text { for } n \geq 2,
$$

then $\left(M_{n}+\psi\left(x_{n}\right)\right) \beta_{n} \leq 1 / 2^{n}$;

(iii) for every $n \geq 1$, we have $\left\|g_{n}\right\|_{\psi} \geq 1 / 2$, with $g_{n}=f_{n} \mathbb{1}_{\left\{\left|f_{n}\right|>\alpha_{n}\right\}}$.

For this, we start with $\beta_{1}$ such that $\left(1+\psi\left(x_{1}\right)\right) \beta_{1}=1 / 2$. Since the norms \|\|$_{\psi}$ and \|\|$_{2}$ are not equivalent on $X_{0}$, there is an $f_{1} \in X_{0}$ with $\left\|f_{1}\right\|_{\psi}=1$ and $\mathbb{P}\left(\left\{\left|f_{1}\right|>\alpha_{1}\right\}\right) \leq \beta_{1}$. Suppose now that $f_{1}, \ldots, f_{n-1}$ and $\beta_{1}, \ldots, \beta_{n-1}$ have been constructed. We then choose $\beta_{n} \leq 1 / 2^{n}$ such that $\left(M_{n}+\psi\left(x_{n}\right)\right) \beta_{n} \leq 1 / 2^{n}$. Since the norms \|\|$_{\psi}$ and \|\|$_{2}$ are not equivalent on $X_{0}$, we can find $f_{n} \in X_{0}$ such that $\left\|f_{n}\right\|_{\psi}=1$ and $\left\|f_{n}\right\|_{2}$ is so small that

$$
\mathbb{P}\left(\left\{\left|f_{n}\right|>\alpha_{n}\right\}\right) \leq \beta_{n} \text {. }
$$

Since $\left\|f_{n}-g_{n}\right\|_{\psi} \leq(1 / a)\left\|f_{n}-g_{n}\right\|_{\infty} \leq \alpha_{n} / a$, we have $\left\|g_{n}\right\|_{\psi} \geq$ $\left\|f_{n}\right\|_{\psi}-\alpha_{n} / a \geq 1 / 2$, and that finishes the construction.

Now, consider

$$
g=\sum_{n=1}^{+\infty}\left|g_{n}\right| .
$$

Set $A_{n}=\left\{\left|f_{n}\right|>\alpha_{n}\right\}$ and

$$
B_{n}=A_{n} \backslash \bigcup_{j>n} A_{j} \quad \text { for } n \geq 1 .
$$

We have $\mathbb{P}\left(\lim \sup A_{n}\right)=0$, because $\sum_{n>1} \mathbb{P}\left(A_{n}\right) \leq \sum_{n>1} \beta_{n}<+\infty$. Now $g$ vanishes off $\bigcup_{n \geq 1} B_{n} \cup\left(\limsup A_{n}\right)$ and $\int_{B_{n}} \psi\left(\left|g_{n}\right|\right) d \mathbb{P} \leq \int_{\Omega} \psi\left(\left|f_{n}\right|\right) d \mathbb{P} \leq 1$. Therefore

$$
\begin{aligned}
\int_{\Omega} \psi\left(\frac{|g|}{4}\right) d \mathbb{P} & =\sum_{n=1}^{+\infty} \int_{B_{n}} \psi\left(\frac{|g|}{4}\right) d \mathbb{P} \\
& \leq \sum_{n=1}^{+\infty} \int_{B_{n}} \frac{1}{2}\left[\psi\left(\frac{\left\|f_{1}\right\|_{\infty}+\cdots+\left\|f_{n-1}\right\|_{\infty}}{2}\right)+\psi\left(\frac{\left|g_{n}\right|}{2}\right)\right] d \mathbb{P}
\end{aligned}
$$

(by convexity of $\psi$ and because $g_{j}=0$ on $B_{n}$ for $j>n$ ) 


$$
\begin{aligned}
& \leq \frac{1}{2} \sum_{n=1}^{+\infty} M_{n} \mathbb{P}\left(A_{n}\right)+\frac{1}{2} \sum_{n=1}^{+\infty} \frac{1}{2^{n}} \int_{B_{n}} \psi\left(\left|g_{n}\right|\right) d \mathbb{P}+\frac{1}{2} \sum_{n=1}^{+\infty} \psi\left(x_{n}\right) \mathbb{P}\left(A_{n}\right) \\
& \leq \frac{1}{2} \sum_{n=1}^{+\infty}\left(M_{n}+\psi\left(x_{n}\right)\right) \beta_{n}+\frac{1}{2} \sum_{n=1}^{+\infty} \frac{1}{2^{n}} \leq 1 .
\end{aligned}
$$

Hence $g \in L^{\psi}(\Omega)$.

It follows that the series $\sum_{n \geq 1} g_{n}$ is weakly unconditionally Cauchy in $X$. Since $\left\|g_{n}\right\|_{\psi} \geq 1 / 2$, it has, by Bessaga-Pełczyński's theorem, a subsequence which is equivalent to the canonical basis of $c_{0}$. The same is true for $\left(f_{n}\right)_{n \geq 1}$ since

$$
\sum_{n=1}^{+\infty}\left\|f_{n}-g_{n}\right\|_{\psi} \leq \frac{1}{a} \sum_{n=1}^{+\infty}\left\|f_{n}-g_{n}\right\|_{\infty} \leq \frac{1}{a} \sum_{n=1}^{+\infty} \alpha_{n}<1 .
$$

That ends the proof.

Of course, the proof shows that the assumption that the norm \|\|$_{\psi}$ is not equivalent to \|\|$_{2}$ can be replaced by the non-equivalence of \|\|$_{\psi}$ to many other norms. We only used the fact that the topology of convergence in measure is not equivalent on $X_{0}$ to the topology defined by \|\|$_{\psi}$.

When we apply this result to the probability space $(G, m)$, we get (see [19, Theorem 2.3]):

TheOREM II.2. Let $\psi$ be as in Theorem II.1 and let $G$ be a compact abelian group. Then, for $\Lambda \subseteq \Gamma=\widehat{G}$, either $L_{\Lambda}^{\psi}$ has cotype 2 , or it contains $c_{0}$. In particular, either $\Lambda$ is a Sidon set and $L_{\Lambda}^{\Psi_{2}}=L_{\Lambda}^{2}$, or $L_{\Lambda}^{\Psi_{2}}$ contains $c_{0}$ (and so it does not have finite cotype).

Proof. Observe that when $L_{\Lambda}^{\psi} \neq L_{\Lambda}^{2}$, the norms \|\|$_{\psi}$ and \|\|$_{2}$ are not equivalent on $X_{0}=\mathcal{P}_{\Lambda}$, the subspace of trigonometric polynomials whose spectrum is contained in $\Lambda$. So the first part follows directly from Theorem II.1. The second one follows from Pisier's characterization of Sidon sets ([35, Théorème 6.2]): $\Lambda$ is a Sidon set if and only if $L_{\Lambda}^{\Psi_{2}}=L_{\Lambda}^{2}$.

Remark. It is proved in [19, Theorem 2.3] that $\Lambda$ is a $\Lambda(\psi)$-set (i.e. $L_{\Lambda}^{\psi}=L_{\Lambda}^{2}$ ) when $L_{\Lambda}^{\Psi_{2}} \subseteq L_{\Lambda}^{\psi} \subseteq L_{\Lambda}^{2}$ and $L_{\Lambda}^{\psi}$ has cotype 2 .

III. Uniform convergence. A function $f \in \mathcal{C}(\mathbb{T})$ is said to have a uniformly convergent Fourier series if $\left\|S_{k}(f)-f\right\|_{\infty} \rightarrow 0$ as $k \rightarrow+\infty$, where

$$
S_{k}(f)=\sum_{j=-k}^{k} \widehat{f}(j) e_{j}
$$


The space $U(\mathbb{T})$ of uniformly convergent Fourier series is the space of all such $f \in \mathcal{C}(\mathbb{T})$. With the norm

$$
\|f\|_{U}=\sup _{k \geq 1}\left\|S_{k}(f)\right\|_{\infty}
$$

$U(\mathbb{T})$ becomes a Banach space.

A set $\Lambda \subseteq \mathbb{Z}$ is said to be a set of uniform convergence (UC-set) if $U_{\Lambda}=\mathcal{C}_{\Lambda}$ as linear spaces. They are then isomorphic as Banach spaces. There exist sets $\Lambda$ which are not $U C$-sets but for which $\mathcal{C}_{\Lambda}$ does not contain $c_{0}$ (for instance, a Rosenthal set which contains arbitrarily long arithmetic progressions [38]). For $U_{\Lambda}$ the situation is different:

TheOrem III.1. If $\Lambda$ is not a $U C$-set, then $U_{\Lambda}$ contains $c_{0}$.

Corollary III.2. If $U_{\Lambda}$ has a finite cotype, then $\Lambda$ is a Sidon set.

Proof. If $U_{\Lambda}$ has a finite cotype, it cannot contain $c_{0}$. Hence $U_{\Lambda}$ is isomorphic to $\mathcal{C}_{\Lambda}$. It follows that $\mathcal{C}_{\Lambda}$ has a finite cotype, and so $\Lambda$ is a Sidon set, by Bourgain-Milman's theorem [3].

REMARK. This result was proved in [19, Theorem 1.2], by adapting the proof of Bourgain and Milman. Now it becomes clear why this proof happened to mimic the original one.

Proof of Theorem III.1. Since $\Lambda$ is not a $U C$-set, there exists a trigonometric polynomial $P_{1} \in \mathcal{C}_{\Lambda}$ with $\left\|P_{1}\right\|_{U}=1$ and $\left\|P_{1}\right\|_{\infty} \leq 1 / 2$. Let $N_{1} \geq 2$ be such that $\widehat{P}_{1}(n)=0$ for $|n| \geq N_{1}$. The spaces $U_{\Lambda \backslash \Lambda \cap\left\{-N_{1}+1, \ldots, 0, \ldots, N_{1}-1\right\}}$ and $\mathcal{C}_{\Lambda \backslash \Lambda \cap\left\{-N_{1}+1, \ldots, 0, \ldots, N_{1}-1\right\}}$ remain non-isomorphic, and so we can find a trigonometric polynomial $P_{2}$ such that $\widehat{P}_{2}(n)=0$ for $|n| \leq N_{1}-1$ with $\left\|P_{2}\right\|_{U}=1$ and $\left\|P_{2}\right\|_{\infty} \leq 1 / 4$. Carrying on this construction, we get a sequence of integers $2 \leq N_{1}<N_{2}<\cdots$ and a sequence of trigonometric polynomials $P_{l} \in \mathcal{C}_{\Lambda}$ such that $\left\|P_{l}\right\|_{U}=1,\left\|P_{l}\right\|_{\infty} \leq 1 / 2^{l}$ and $\widehat{P}_{l}(n)=0$ for $n \notin\left\{ \pm N_{l-1}, \ldots, \pm\left(N_{l}-1\right)\right\}$.

Now, fix an integer $L \geq 1$ and a sequence $a_{1}, \ldots, a_{L}$ of complex numbers. For each $k \geq 1$, let $l_{k}$ be such that $N_{l_{k}} \leq k<N_{l_{k}+1}$. When $L \geq l_{k}+1$, we have

$$
\begin{aligned}
\left\|S_{k}\left(\sum_{l=1}^{L} a_{l} P_{l}\right)\right\|_{\infty} & \leq\left\|\sum_{l=1}^{l_{k}} a_{l} P_{l}\right\|_{\infty}+\left\|a_{l_{k}+1} S_{k}\left(P_{l_{k}+1}\right)\right\|_{\infty} \\
& \leq \max _{1 \leq j \leq l_{k}}\left|a_{j}\right| \sum_{l=1}^{l_{k}}\left\|P_{l}\right\|_{\infty}+\left|a_{l_{k}+1}\right|\left\|P_{l_{k}+1}\right\|_{U} \\
& \leq 2 \max \left\{\left|a_{1}\right|, \ldots,\left|a_{l_{k}}\right|,\left|a_{l_{k}+1}\right|, \ldots,\left|a_{L}\right|\right\} .
\end{aligned}
$$

The inequality $\left\|S_{k}\left(\sum_{l=1}^{L} a_{l} P_{l}\right)\right\|_{\infty} \leq 2 \max \left\{\left|a_{1}\right|, \ldots,\left|a_{l_{k}}\right|,\left|a_{l_{k}+1}\right|, \ldots,\left|a_{L}\right|\right\}$ remains trivially true for $L \leq l_{k}$, because in this case $S_{k}\left(\sum_{l=1}^{L} a_{l} P_{l}\right)=$ 
$\sum_{l=1}^{L} a_{l} P_{l}$. Therefore we get

$$
\left\|\sum_{l=1}^{L} a_{l} P_{l}\right\|_{U} \leq 2 \max \left\{\left|a_{1}\right|, \ldots,\left|a_{L}\right|\right\} .
$$

It follows that the series $\sum_{l>1} P_{l}$ is weakly unconditionally Cauchy. Since it is obviously not convergent, $U_{\Lambda}$ contains a subspace isomorphic to $c_{0}$ by Bessaga-Pełczyński's theorem (see [6, pp. 44-45, Theorems 6 and 8]).

REMARK 1. There is a stronger notion of $C U C$-set. $\Lambda \subseteq \mathbb{Z}$ is a $C U C$-set if

$$
\left\|\sum_{j=k_{1}}^{k_{2}} \widehat{f}(j) e_{j}-f\right\|_{\infty} \underset{\substack{k_{1} \rightarrow-\infty \\ k_{2} \rightarrow+\infty}}{\stackrel{\longrightarrow}{\longrightarrow}} 0 \text { for every } f \in \mathcal{C}_{\Lambda} .
$$

Obviously, for subsets of $\mathbb{N}$, the two notions coincide. Theorem III.1 is not valid for $C U C$-sets: let $H$ be an Hadamard lacunary sequence. Then $\Lambda=$ $H-H$ is not a $C U C$-set (Fournier [8]), but it is $U C$ and Rosenthal, so that $U_{\Lambda}=\mathcal{C}_{\Lambda}$ does not contain $c_{0}$.

However, it is not known whether $\mathcal{C}_{\Lambda_{1} \cup \Lambda_{2}}$ lacks $c_{0}$ whenever this is true for $\mathcal{C}_{\Lambda_{1}}$ and $\mathcal{C}_{\Lambda_{2}}$. If we replace the space $\mathcal{C}(G)$ by $U(\mathbb{T})$, the answer is in the negative. Indeed, J. Fournier shows $([8])$, completing Soardi and Travaglini's work [43], that there exist two $U C$-sets $\Lambda_{1}, \Lambda_{2} \subseteq \mathbb{Z}$ which are Rosenthal sets but $\Lambda_{1} \cup \Lambda_{2}=H+H-H$ is not $U C$. Therefore $U_{\Lambda_{1}}=\mathcal{C}_{\Lambda_{1}}$ and $U_{\Lambda_{2}}=\mathcal{C}_{\Lambda_{2}}$ do not contain $c_{0}$, though $U_{\Lambda_{1} \cup \Lambda_{2}}$ contains $c_{0}$.

REMARK 2. $U C$-sets $\Lambda$ for which $\mathcal{C}_{\Lambda}$ contains $c_{0}$ are constructed in [24].

REMARK 3. We stated Theorem III.1 for uniform convergence because it is the classical case. Actually, J. Fournier ([8, p. 72]) and S. Hartman ([13, p. 107]) introduced the space $L^{1}-U C$ as the set of all $f \in L^{1}(\mathbb{T})$ for which $\left\|S_{k}(f)-f\right\|_{1} \rightarrow 0$ as $k \rightarrow+\infty$. It is normed by $\|f\|_{U L^{1}}=\sup _{k>1}\left\|S_{k}(f)\right\|_{1}$. We call $\Lambda$ an $L^{1}-U C$-set if $\left(L^{1}-U C\right)_{\Lambda}=L_{\Lambda}^{1}$. The same proof as above shows that if $\left(L^{1}-U C\right)_{\Lambda} \neq L_{\Lambda}^{1}$, then $\left(L^{1}-U C\right)_{\Lambda}$ contains $c_{0}$. More generally, let $\Lambda \subseteq \mathbb{Z}$ and let $X$ be a Banach space contained, as a linear subspace, in $L^{1}(\mathbb{T})$ such that the linear space generated by $X \cap A$ is dense in $X$. We can define $X-U C$ in an obvious way, and we have: if $X-U C$ is not isomorphic to $X$, then it contains $c_{0}$.

We give another consequence of Theorem III.1. Recall (see [30]) that $\Lambda \subseteq \Gamma$ is a Riesz set if every measure with spectrum in $\Lambda$ is absolutely continuous with respect to the Haar measure (in short, $\mathcal{M}_{\Lambda}=L_{\Lambda}^{1}$ ).

Corollary III.3. If $U_{\Lambda}$ does not contain $c_{0}$, then $\Lambda$ is a Riesz set.

Proof. If $U_{\Lambda} \nsupseteq c_{0}$, then $U_{\Lambda}=\mathcal{C}_{\Lambda}$, by Theorem III.1, and so $\mathcal{C}_{\Lambda} \nsupseteq c_{0}$. It then follows that $\Lambda$ is a Riesz set (F. Lust-Piquard [25], her first Thé- 
orème 3.1). Let us recall why. For $\mu \in \mathcal{M}_{\Lambda}$, the convolution operator $C_{\mu}$ : $f \in \mathcal{C}(G) \mapsto f * \mu \in \mathcal{C}_{\Lambda} \subseteq \mathcal{C}(G)$ is weakly compact, because $\mathcal{C}(G)$ has Pełczyński's property $(V)$ and $\mathcal{C}_{\Lambda} \nsupseteq c_{0}$. Its adjoint operator $\nu \in \mathcal{M}(G) \mapsto$ $\nu * \mu \in \mathcal{M}_{\Lambda}$ is also weakly compact. Hence, if $\left(K_{j}\right)_{j}$ is an approximate unit for the convolution, there is a sequence $\left(j_{n}\right)_{n}$ such that $K_{j_{n}} * \mu$ is weakly convergent. Since $K_{j} * \mu$ converges weak-star to $\mu$, it follows that $\mu \in L_{\Lambda}^{1}$.

Remark. Another proof can be given, without using Theorem III.1, but using the fact that $U(\mathbb{T})$ has Pełczyński's property $(V)$ (Saccone [42, Theorem 2.2]; for $U_{\mathbb{N}}(\mathbb{T})$, see Bourgain [1, Lemme 2 and Lemme 3], and Saccone [41, Theorem 4.1]). Then, as before, $K_{j_{n}} * \mu$ is weakly convergent, in $U(\mathbb{T})^{*}$ this time. So there are convex combinations which converge in the norm of $U(\mathbb{T})^{*}$. But then they converge in the norm of $U_{\mathbb{N}}(\mathbb{T})^{*}$, and so $u \in L^{1}(G)$ (see D. Oberlin [33, p. 310]). Note that Oberlin's argument (as well as Bourgain's) depends on Carleson's theorem (via [47]).

IV. Invariant means and Hilbert sets. An invariant mean $M$ on $L^{\infty}(G)$ is a continuous linear functional on $L^{\infty}(G)$ such that $M(\mathbb{1})=\|M\|$ $=1$ and $M\left(f_{x}\right)=M(f)$ for every $f \in L^{\infty}(G)$. The Haar measure $m$ defines an invariant mean, and W. Rudin ([40]) showed that, for infinite compact abelian groups $G$, there always exist other invariant means on $L^{\infty}(G)$. A function $f \in L^{\infty}(G)$ has a unique invariant mean if $M(f)=\widehat{f}(0)$ for every invariant mean $M$ on $L^{\infty}(G)$. Every continuous function (or, even, every Riemann-integrable function: [39, p. 38] or [44]) has a unique invariant mean.

Definition IV.1. A subset $\Lambda$ of $\Gamma=\widehat{G}$ is called a Lust-Piquard set if $\gamma f$ has a unique invariant mean for every $f \in L_{\Lambda}^{\infty}$ and every $\gamma \in \Gamma$.

In other words, $\Lambda$ is a Lust-Piquard set if for every invariant mean $M$ on $L^{\infty}(G)$ and every $f \in L_{\Lambda}^{\infty}$, one has

$$
M(\gamma f)=\widehat{f}(-\gamma)
$$

In [26] (and then in [21]; see also [28]), F. Lust-Piquard called them totally ergodic sets. We use a different name because J. Bourgain ([2, 2.I, p. 206]), used the term "ergodic set" for another property (see also [24]).

Note that it is required that the invariant means agree on $\bigcup_{\gamma \in \Gamma} L_{\Lambda-\gamma}^{\infty}$, and not only on $L_{\Lambda}^{\infty}$, because the invariant means may coincide on $L_{\Lambda}^{\infty}$ for trivial reasons; for instance, all the invariant means are equal to 0 on $L_{2 \mathbb{Z}+1}^{\infty}$ (since $f(x+1 / 2)=-f(x)$ for $\left.f \in L_{2 \mathbb{Z}+1}^{\infty}\right)$. It is clear that if $\Lambda$ is a Lust-Piquard set, then $\Lambda-\gamma$ is also a Lust-Piquard set for every $\gamma \in \Gamma$.

It is obvious that every Rosenthal set is a Lust-Piquard set (since every continuous function has a unique invariant mean), and it is shown in [21] 
that every Lust-Piquard set is a Riesz set. On the other hand, Y. Katznelson (see [39, pp. 37-38]) proved that $\mathbb{N}$ is not a Lust-Piquard set.

F. Lust-Piquard ([27, Theorems 2 and 4$])$ showed that $\Lambda=\mathbb{P} \cap(5 \mathbb{Z}+2)$, where $\mathbb{P}$ is the set of prime numbers, is totally ergodic (a Lust-Piquard set in our terminology) although $\mathcal{C}_{\Lambda}$ contains $c_{0}$.

In the following theorem, we give another example of such a situation. Let us recall that $H \subseteq \mathbb{Z}$ is a Hilbert set if there exist two sequences of integers $\left(p_{n}\right)_{n \geq 1}$ and $\left(q_{n}\right)_{n \geq 1}$, with $q_{n} \neq 0$, such that

$$
H=\bigcup_{n \geq 1}\left\{p_{n}+\sum_{k=1}^{n} \varepsilon_{k} q_{k} ; \varepsilon_{1}, \ldots, \varepsilon_{n}=0 \text { or } 1\right\} .
$$

It is shown in [22, Theorem 2] that $\mathcal{C}_{H}$ contains $c_{0}$ when $H$ is a Hilbert set.

Theorem IV.2. There exists a Hilbert set $H \subseteq \mathbb{N}$ which is a LustPiquard set.

We begin with a lemma, which is implicit in [27, proof of Theorem 4].

Lemma IV.3. The family of Lust-Piquard sets in $\Gamma$ is localizable for the Bohr topology.

Let us recall that the Bohr topology of a discrete abelian group $\Gamma$ is the topology of pointwise convergence, when $\Gamma$ is seen as a subset of $\mathcal{C}(G)$; it is also the natural topology on $\Gamma$ as a subset of the dual group of $G_{\mathrm{d}}$, the group $G$ with the discrete topology. A class $\mathcal{F}$ of subsets of $\Gamma$ is localizable for the Bohr topology if $\Lambda \in \mathcal{F}$ whenever for every $\gamma \in \Gamma$ there is a neighbourhood $V_{\gamma}$ of $\gamma$ for the Bohr topology such that $\Lambda \cap V_{\gamma} \in \mathcal{F}$. This notion is due to Y. Meyer ([30]).

For the sake of completeness, we give a proof.

Proof of Lemma IV.3. We are going to prove that if $V_{\gamma}$ is a neighbourhood of $\gamma \in \Gamma$ such that $\Lambda \cap V_{\gamma}$ is a Lust-Piquard set, then $\bar{\gamma} f$ has a unique invariant mean for every $f \in L_{\Lambda}^{\infty}$, and that will prove the lemma.

By the regularity of the algebra $L^{1}\left(G_{\mathrm{d}}\right)=\ell_{1}(G)=\mathcal{M}_{\mathrm{d}}(G)$, there exists a discrete measure $\nu \in \mathcal{M}_{\mathrm{d}}(G)$ such that $\widehat{\nu}(\gamma)=1$ and $\widehat{\nu}=0$ outside $V_{\gamma}$. Since $(\bar{\gamma} f) *(\bar{\gamma} \nu) \in L_{\left(\Lambda \cap V_{\gamma}\right)-\gamma}^{\infty}$, and since $\left(\Lambda \cap V_{\gamma}\right)-\gamma$ is a Lust-Piquard set, we have

$$
M((\bar{\gamma} f) *(\bar{\gamma} \nu))=[(\bar{\gamma} f) *(\bar{\gamma} \nu)]^{\wedge}(0)=\widehat{f}(\gamma) \widehat{\nu}(\gamma)=\widehat{f}(\gamma) .
$$

But $\bar{\gamma} \nu$ is a discrete measure, and for every discrete measure $\mu$ we have

$$
M(\mu * g)=M(g) \widehat{\mu}(0)
$$

for every $g \in L^{\infty}(G)$ and every invariant mean $M$. This is so since if $\mu=\sum_{k} a_{k} \delta_{x_{k}}$ with $\sum_{k}\left|a_{k}\right|<+\infty$, we have $M(\mu * g)=\sum_{k} a_{k} M\left(g_{x_{k}}\right)=$ $\sum_{k} a_{k} M(g)$.

Hence $M(\bar{\gamma} f)=\widehat{f}(\gamma)$, as required. 
Proof of Theorem IV.2. We are going to construct a Hilbert set $H \subseteq \mathbb{N}$ which is discrete in $\mathbb{Z}$ for the Bohr topology. For such a set, there is, for every $k \in \mathbb{Z}$, some Bohr neighbourhood $V_{k}$ of $k$ such that $H \cap V_{k}$ is finite. Therefore, we have $L_{H \cap V_{k}}^{\infty}=\mathcal{C}_{H \cap V_{k}}$, and so $H \cap V_{k}$ is a Lust-Piquard set.

Let $\left(d_{n}\right)_{n \geq 0}$ be a strictly increasing sequence of positive integers such that

$$
d_{n} \mid d_{n+1}, \quad n \geq 0, \quad \sum_{n=0}^{+\infty} \frac{2^{n+1}}{d_{n}}<1 .
$$

For every $k \in \mathbb{Z}$, consider

$$
V(k)=k+d_{|k|} \mathbb{Z},
$$

which is a Bohr neighbourhood of $k$.

Now, we are going to show that for every $n \geq 0$ we can choose an integer $r_{n} \in\left\{0,1, \ldots, d_{n}-1\right\}$ such that if

$$
H_{n}=d_{n}+r_{n}+\left\{\sum_{l=0}^{n-1} \varepsilon_{l} d_{l} ; \varepsilon_{l}=0 \text { or } 1\right\},
$$

then $H_{p} \cap V(k)=\emptyset$ for every $k \in \mathbb{Z}$ and every $p>|k|$. The set $H=\bigcup_{n \geq 0} H_{n}$ will be the required set.

We are going to do this by induction. First, we may choose an arbitrary $r_{0} \in\left\{0,1, \ldots, d_{0}-1\right\}$, and we set $H_{0}=\left\{d_{0}+r_{0}\right\}$. Suppose now that we have found $r_{1}, \ldots, r_{p-1}$ such that the previous conditions are satisfied:

$$
H_{j} \cap V(k)=\emptyset \quad \text { for } 1 \leq j \leq p-1,|k|<j .
$$

To find $r_{p}$, note that $m \in H_{p} \cap V(k)$ if and only if

$$
m \in k+d_{|k|} \mathbb{Z}
$$

and there exist $\varepsilon_{0}, \varepsilon_{1}, \ldots, \varepsilon_{p-1} \in\{0,1\}$ such that

$$
m=d_{p}+r_{p}+\sum_{l=0}^{p-1} \varepsilon_{l} d_{l} .
$$

Since, for $0 \leq l<p$, one has $d_{l}\left|d_{l+1}\right| \ldots \mid d_{p}$, conditions (1) and (2) are equivalent to $r_{p} \equiv 0\left(\bmod d_{0}\right)$ for $k=0$, and for $1 \leq l=|k|<p$ to

$$
k \equiv r_{p}+\sum_{j=0}^{|k|-1} \varepsilon_{j} d_{j}\left(\bmod d_{|k|}\right) .
$$

For each such $k(0 \leq|k|<p)$, there are

$$
\frac{d_{p}}{d_{|k|}} \cdot 2^{|k|}
$$


possible choices for $r_{p}$. As

$$
\frac{d_{p}}{d_{0}}+2 \sum_{l=1}^{p-1} 2^{l} \frac{d_{p}}{d_{l}} \leq \frac{d_{p}}{d_{0}}+2 \sum_{l=1}^{+\infty} 2^{l} \frac{d_{p}}{d_{l}}<d_{p}
$$

by hypothesis, we can find an $r_{p} \in\left\{0,1, \ldots, d_{p}-1\right\}$ such that the set $H_{p}$ constructed from it satisfies $H_{p} \cap V(k)=\emptyset$ for $|k|<p$. That ends the proof.

REMARK 1. A subclass of Hilbert sets are the IP-sets, i.e. sets $F$ for which there exists a sequence $\left(p_{n}\right)_{n \geq 1}$ of integers such that

$$
F=\left\{\sum_{k=1}^{n} \varepsilon_{k} p_{k} ; \varepsilon_{1}, \ldots, \varepsilon_{n}=0 \text { or } 1, n \geq 1\right\} .
$$

Question. Does there exist an $I P$-set $F$ which is a Lust-Piquard set?

Every point of an $I P$-set $F$ is non-isolated in $F$ (see [10, Theorem 2.19]; note that every point of an $I P$-set is inside the translation by this point of a sub- $I P$-set). Therefore we cannot use an argument similar to that of the previous theorem. Hilbert sets and $I P$-sets are different in several ways. For instance, every set $\Lambda \subseteq \mathbb{Z}$ which has a positive uniform density contains a Hilbert set ([14, Theorem 11.11], [22, Theorem 4]), but not necessarily an $I P$-set ([14, Theorem 11.6], [32, p. 151]). Another difference is that $\mathcal{C}_{\Lambda}$ never has the Unconditional Metric Approximation Property if $\Lambda \subseteq \mathbb{Z}$ is an $I P$-set ([23, Proposition 11]), but can have this property when $\Lambda$ is a Hilbert set ([23, Theorem 10]).

REMARK 2. Let $\mathcal{F}$ be a class of subsets of $\Gamma$ which contains all the finite sets and which is localizable for the Bohr topology. It follows from the proof of Theorem IV.2 that such a class must contain some Hilbert sets. In particular $\mathcal{F}$ has to contain sets $\Lambda$ such that $\Lambda$ contains parallelepipeds of arbitrarily large dimensions. Note that this last assertion is actually implicit in [27]. Indeed, by Dirichlet's theorem $\sum_{n \in \mathbb{P} \cap(5 \mathbb{Z}+2)} 1 / n=+\infty$, and by [31, Corollary 2], we have $\sum_{n \in \Lambda} 1 / n<+\infty$ when $\Lambda$ does not contain parallelepipeds of arbitrarily large dimensions. It is known that the sets belonging to the following classes cannot contain parallelepipeds of arbitrarily large dimensions:

(a) $\Lambda(p)-$ sets (see [31, Theorem 3] and [9, Theorem 4]).

(b) $U C$-sets $([9$, Theorem 4]).

(c) $p$-Sidon sets $([15$, Lemma 1$])$.

(d) Stationary sets ([18, Proposition 2.5]). 
(e) $q$-Rider sets (see [24] or [19] for the definition). Note that, for $1 \leq$ $q<4 / 3, q$-Rider sets are $p$-Sidon sets for every $p>q /(2-q)$ (see [20]), and so the result is in (c). For $4 / 3 \leq q<2$, there is no explicit published proof of that, and therefore we shall give one in Proposition IV.4, after this Remark.

Hence these classes are not localizable for the Bohr topology.

Remark 2 shows that there is no hope to construct sets of the above classes by way of localization.

Proposition IV.4. If $\Lambda$ is a q-Rider set, $1 \leq q<2$, then $\Lambda$ cannot contain parallelepipeds of arbitrarily large dimensions.

Proof. A Sidon set (with constant less than 10, say) inside a parallelepiped $P$ of size $2^{n}$ cannot contain more than $C n \log n$ elements ([16, Chapter 6, $\S 3$, Theorem 5, p. 71]), whereas if $P$ were contained in a $q$-Rider set, it should contain a quasi-independent (hence Sidon with constant less than 10) set of size at least $C 2^{\varepsilon n}$, with $\varepsilon=(2-q) / q([36]$ or [37, Teorema 2.3]).

Note that another proof of Proposition IV.4 is implicit in [15]. Indeed the proof given in $[15$, Lemma 1] that $p$-Sidon sets share this property only uses the fact, proved in [4, Eq. (9)], that if $\Lambda$ is a $p$-Sidon set, then, with $\alpha=2 p /(3 p-2)$, there is a constant $C>0$ such that $\|f\|_{r} \leq C \sqrt{r}\|\widehat{f}\|_{\alpha}$ for all $r \geq 2$ (equivalently: $\|f\|_{\Psi_{2}} \leq C^{\prime}\|\widehat{f}\|_{\alpha}$ ) for every $f \in \mathcal{C}_{\Lambda}$. Now the fourth-named author proved that these inequalities characterize $p$-Rider sets ([36]; see also [37, Teorema 2.3]).

V. Complemented subspaces. Since $\Lambda$ is a Rosenthal set if $L_{\Lambda}^{\infty}=\mathcal{C}_{\Lambda}$, it is natural to ask whether $\Lambda$ is a Rosenthal set if there exists a projection from $L_{\Lambda}^{\infty}$ onto $\mathcal{C}_{\Lambda}$. We have not been able to answer this, even if this projection were to have norm 1 (see [12], where the condition that the space does not contain $\ell_{1}$ is crucial), but we have a partial result. Recall that it is not known whether $\mathcal{C}_{\Lambda} \nsupseteq c_{0}$ implies that $\Lambda$ is a Rosenthal set.

TheOREM V.1. Let $\Lambda \subseteq \Gamma$ be such that there exists a surjective projection $P: L_{\Lambda}^{\infty} \rightarrow \mathcal{C}_{\Lambda}$. Then $\mathcal{C}_{\Lambda}$ does not contain $c_{0}$. Moreover, every Riemannintegrable function in $L_{\Lambda}^{\infty}$ is actually in $\mathcal{C}_{\Lambda}$.

Recall that a function $h: G \rightarrow \mathbb{C}$ is Riemann-integrable if it is bounded and almost everywhere continuous. Actually, the last assertion of the proposition means that every element of $L_{\Lambda}^{\infty}$ which contains a Riemann-integrable function contains also a continuous one.

Proof. (1) By [22, Proposition 14], if $\mathcal{C}_{\Lambda}$ contains $c_{0}$, there is a sequence $\left(f_{n}\right)_{n \geq 1}$ in $\mathcal{C}_{\Lambda}$ which is equivalent to the canonical basis of $c_{0}$, and whose 
$w^{*}$-linear span $F$ in $L_{\Lambda}^{\infty}$ is isomorphic to $\ell_{\infty}$. The restriction $\left.P\right|_{F}$ is a projection from $F$ onto a subspace of $\mathcal{C}_{\Lambda}$ which contains $E=\overline{\operatorname{span}}\left\{f_{n} ; n \geq 1\right\}$.

Observe that $E$ is a separable subspace of $\mathcal{C}_{\Lambda}$. So there exists a countable subset $\Lambda_{1} \subseteq \Lambda$ such that $E \subseteq \mathcal{C}_{\Lambda_{1}}$. Moreover, there exists a countable subgroup $\Gamma_{0} \subseteq \Gamma$ such that $\Lambda_{1}$ is contained in $\Gamma_{0}$. Taking $\Lambda_{0}=\Lambda \cap \Gamma_{0}$, we have $E \subseteq \mathcal{C}_{\Lambda_{0}}$, and $\mathcal{C}_{\Lambda_{0}}$ is a separable space.

The set $\Gamma_{0}$ being a subgroup, there exists a measure $\mu$ on $G$ whose Fourier transform is $\widehat{\mu}=\mathbb{1}_{\Gamma_{0}}$. The map $f \mapsto f * \mu$ gives a projection from $\mathcal{C}_{\Lambda}$ onto $\mathcal{C}_{\Lambda_{0}}$, and Sobczyk's theorem gives a projection from $\mathcal{C}_{\Lambda_{0}}$ onto $E$. So there exists a projection from $F \simeq \ell_{\infty}$ onto $E \simeq c_{0}$, which is a contradiction.

(2) We first assume that the group $G$ is metrizable, so that $\mathcal{C}(G)$ is separable. Let $R I_{\Lambda}$ be the subspace of $L_{\Lambda}^{\infty}$ consisting of the Riemann-integrable functions (more precisely: the elements of $L_{\Lambda}^{\infty}$ which have a Riemann-integrable representative).

Consider the restriction of $P$ to $R I_{\Lambda}$. For $f \in R I$, the set

$$
\left\{x \mapsto \xi\left(f_{x}\right) ; \xi \in L^{\infty}(G)^{*},\|\xi\| \leq 1\right\}
$$

is stable $([46$, Theorem $(15-6)(\mathrm{c})])$. Let $\mu \in\left(\mathcal{C}_{\Lambda}\right)^{*}$, and set $\varphi(x, y)=$ $\left(P^{*} \mu_{y}\right)\left(f_{x}\right)$ for $x, y \in G$. The map $x \in G \mapsto f_{x} \in L^{\infty}(G)$ is scalarly measurable ([45, Theorem 16]) and $y \mapsto P^{*} \mu_{y}$ is continuous for the $w^{*}$ topology. Moreover $\left\{x \mapsto\left(P^{*} \mu_{y}\right)\left(f_{x}\right) ; y \in G\right\}$ is stable, so by [46, Theorem $(10-2-1)], \varphi$ is measurable. Measurability refers here to the completion of the product measure $m \otimes m$ on $G \times G$, so in order to deduce that the map $x \in G \mapsto \varphi(x, x)=\left(P^{*} \mu_{x}\right)\left(f_{x}\right)$ is measurable, we need the following lemma (note that our $\varphi$ is bounded).

Lemma V.2. Let $G$ be a metrizable compact abelian group, and $\varphi: G \times$ $G \rightarrow \mathbb{C}$ a function such that:

(a) $\varphi \in \mathcal{L}^{\infty}(G \times G)$;

(b) the map $y \mapsto \varphi(x, y)$ is continuous for every $x \in G$.

Then the map $x \mapsto \varphi(x, x)$ is measurable.

Proof. $G$ being metrizable, there exists a bounded sequence $\left(f_{n}\right)_{n}$ in $L^{1}(G)$ such that

$$
g(0)=\lim _{n \rightarrow \infty} \int_{G} f_{n} g d m \quad \text { for every } g \in \mathcal{C}(G) .
$$

This sequence $\left(f_{n}\right)_{n}$ represents an approximate identity.

For every $n$, the function $(x, y) \mapsto f_{n}(x-y) \varphi(x, y)$ is integrable in $G \times G$. Define

$$
F_{n}(x)=\int_{G} f_{n}(x-y) \varphi(x, y) d m(y)=\int_{G} f_{n}(t) \varphi(x, x-t) d m(t) .
$$


By Fubini's theorem $F_{n}$ is defined almost everywhere, and is integrable. So $F_{n}$ is measurable for every $n$. The lemma follows since, by (3),

$$
\varphi(x, x)=\lim _{n \rightarrow \infty} F_{n}(x) \quad \text { for every } x \in G .
$$

The fact that the map $x \in G \mapsto\left(P^{*} \mu_{x}\right)\left(f_{x}\right)=\left\langle\mu,\left[P\left(f_{x}\right)\right]_{-x}\right\rangle$ is measurable means, since $\mu$ is arbitrary, that $x \mapsto\left[P\left(f_{x}\right)\right]_{-x} \in \mathcal{C}_{\Lambda}$ is scalarly measurable. Since we have assumed that $\mathcal{C}(G)$ is separable, this map is strongly measurable, by Pettis's measurability theorem ([7, II, $\S 1$, Theorem 2]). Now we showed at the beginning of the proof that $\mathcal{C}_{\Lambda}$ does not contain $c_{0}$; so a result of J. Diestel [5] (see [7, II, §3, Theorem 7]) says that this map is Pettis-integrable, which means that if we define $Q f$ using

$$
\langle Q f, \mu\rangle=\int_{G}\left\langle f_{x}, P^{*}\left(\mu_{x}\right)\right\rangle d x
$$

for every $\mu \in\left(\mathcal{C}_{\Lambda}\right)^{*}$, then $Q$ maps $R I_{\Lambda}$ into $\mathcal{C}_{\Lambda}$, and not only into its bidual (see the definition of Pettis-integrability in [7, II, §3, p. 53, Definition 2], or in [46, Definition (4-2-1)]).

Thus $Q$ is a projection from $R I_{\Lambda}$ onto $\mathcal{C}_{\Lambda}$ such that $Q\left(f_{x}\right)=(Q f)_{x}$ for every $f \in R I_{\Lambda}$ and every $x \in G$.

We want to prove that $Q f=f$ for every $f \in R I_{\Lambda}$, and for that we have to see that $\widehat{Q f}(\gamma)=\widehat{f}(\gamma)$ for every $\gamma \in \Gamma$. But it suffices to show that $\widehat{Q f}(0)=\widehat{f}(0)$, since, after replacing $\Lambda$ by $\Lambda-\gamma$ and $Q$ by $Q_{\gamma}$ : $L_{\Lambda-\gamma}^{\infty} \rightarrow \mathcal{C}_{\Lambda-\gamma}$, with $Q_{\gamma}(g)=\bar{\gamma} Q(\gamma g)$, we then get, for $f \in R I_{\Lambda}$ with $g=\bar{\gamma} f$,

$$
\widehat{Q f}(\gamma)=[\bar{\gamma}(Q f)]^{\wedge}(0)=\widehat{Q_{\gamma}} g(0)=\widehat{g}(0)=\widehat{(\bar{\gamma} f)}(0)=\widehat{f}(\gamma)
$$

So, let $f \in R I_{\Lambda}$. Every Riemann-integrable function has a unique invariant mean ([39, Lemma 7], [44]); hence there are ([39, Proposition, p. 38], or [26, Proposition 1]) convex combinations $\sum_{k \in I_{n}} c_{n, k} f_{x_{n, k}}, c_{n, k}>0$, $\sum_{k \in I_{n}} c_{n, k}=1$, of translates of $f$ which converge in norm to the constant function $\widehat{f}(0) \mathbb{1}$. We have

$$
Q\left(\sum_{k \in I_{n}} c_{n, k} f_{x_{n, k}}\right) \underset{n \rightarrow+\infty}{\longrightarrow} Q[\widehat{f}(0) \mathbb{1}]=\widehat{f}(0) \mathbb{1} .
$$

But $Q\left(\sum_{k \in I_{n}} c_{n, k} f_{x_{n, k}}\right)=\sum_{k \in I_{n}} c_{n, k}(Q f)_{x_{n, k}}$, and its Fourier coefficient at 0 is

$$
\sum_{k \in I_{n}} c_{n, k} \widehat{Q f}(0)=\widehat{Q f}(0) .
$$

Therefore $\widehat{Q f}(0)=\widehat{f}(0)$.

(3) In order to finish the proof, we have to explain why we may assume that $G$ is metrizable. 
Let $\Lambda$ be as in the theorem, and $f \in R I_{\Lambda}$. As explained in the proof of the first part of the theorem, there exists a countable subgroup $\Gamma_{0} \subseteq \Gamma$ such that $f \in R I_{\Lambda_{0}}$ for $\Lambda_{0}=\Lambda \cap \Gamma_{0}$, and there exists a projection from $L_{\Lambda_{0}}^{\infty}$ onto $\mathcal{C}_{\Lambda_{0}}$.

Let $H$ be the annihilator of $\Gamma_{0}$; that is, $H$ is the following closed subgroup of $G$ :

$$
H=\Gamma_{0}^{\perp}=\left\{x \in G ; \gamma(x)=1, \forall \gamma \in \Gamma_{0}\right\} .
$$

The quotient group $G / H$ is metrizable since its dual group $\Gamma_{0}$ is countable. Let $\pi_{H}$ denote the quotient map from $G$ onto $G / H$. It is known that that the map $g \mapsto g \circ \pi_{H}$ gives an isometry from $L_{\Lambda_{0}}^{\infty}(G / H)$ onto $L_{\Lambda_{0}}^{\infty}(G)$ sending $\mathcal{C}_{\Lambda_{0}}(G / H)$ onto $\mathcal{C}_{\Lambda_{0}}(G)$.

In order to finish our reduction to the metrizable case we only have to see that this isometry sends $R I_{\Lambda_{0}}(G / H)$ onto $R I_{\Lambda_{0}}(G)$. It is easy to see, via the map $g \mapsto g \circ \pi_{H}$, that having a Riemann-integrable function $g: G / H \rightarrow \mathbb{C}$ is the same as having a Riemann-integrable function $g: G \rightarrow \mathbb{C}$ with the property $g(x+h)=g(x)$ for every $x \in G$ and every $h \in H$. Therefore the above isometry sends $R I_{\Lambda_{0}}(G / H)$ into $R I_{\Lambda_{0}}(G)$. The surjectivity of this map is a consequence of the following proposition:

Proposition V.3. Let $f: G \rightarrow \mathbb{C}$ be a Riemann-integrable function such that $\widehat{f}(\gamma)=0$ for every $\gamma \in \Gamma \backslash \Gamma_{0}$. Then there exists a Riemann-integrable function $g: G \rightarrow \mathbb{C}$ such that:

(a) $f=g$ almost everywhere;

(b) $g(x)=g(x+h)$ for all $x \in G$ and $h \in H$.

Proof. We can and will assume that $f$ is in fact real-valued. Take an increasing sequence $\left(K_{n}\right)_{n}$ of compact subsets of $G$ such that if $B=\bigcup_{n} K_{n}$, then:

(i) $f$ is continuous at every point of $B$;

(ii) $m(G \backslash B)=0$.

Using the compactness of $K_{n}$ and the continuity of $f$ on $B$, one can find a neighbourhood $W_{n}$ of 0 such that

$$
|f(x)-f(x+y)| \leq \frac{1}{n} \quad \text { for every } x \in K_{n} \text { and every } y \in W_{n} .
$$

Let $\left(V_{n}\right)_{n}$ be a decreasing sequence of open symmetric neighbourhoods of 0 such that $V_{n}+V_{n} \subseteq W_{n}$ for every $n$. For every $n$, define $f_{n}$ as

$$
f_{n}(x)=\frac{1}{m\left(V_{n}\right)} \int_{V_{n}} f(x-y) d m(y), \quad x \in G .
$$

Then $f_{n}$ is a continuous function since it is the convolution of $f$ and

$$
\psi_{n}=\frac{1}{m\left(V_{n}\right)} \mathbb{1}_{V_{n}} .
$$


We also have

$$
\widehat{f}_{n}(\gamma)=\widehat{f}(\gamma) \widehat{\psi}_{n}(\gamma)=0 \quad \text { for all } \gamma \in \Gamma \backslash \Gamma_{0}
$$

Then the continuous function $f_{n}$ only depends on the classes in $G / H$; that is,

$$
f_{n}(x)=f_{n}(x+h) \quad \text { for all } x \in G, h \in H \text { and } n \text {. }
$$

Define

$$
g(x)=\frac{1}{2}\left(\limsup _{n \rightarrow \infty} f_{n}(x)+\liminf _{n \rightarrow \infty} f_{n}(x)\right), \quad x \in G .
$$

It is clear that $g(x)=g(x+h)$ for all $x \in G$ and $h \in H$. Since $V_{n} \subseteq W_{n}$, we deduce from (4) that $\left|f_{n}(x)-f(x)\right| \leq 1 / n$ for all $x \in K_{n}$. If $x \in B=$ $\bigcup_{n} K_{n}$, then there exists $N$ such that $x \in K_{n}$ for all $n \geq N$. Therefore $\left|f_{n}(x)-f(x)\right| \leq 1 / n$ for all $n \geq N$, and $g(x)=f(x)$. So $f=g$ almost everywhere.

In order to finish the proof we are going to see that every point of $B$ is a point of continuity of $g$, and so $g$ is Riemann-integrable. Let $x$ be in $B$. Given $\varepsilon>0$, there exists $N$ such that $1 / N \leq \varepsilon$ and $x \in K_{n}$ for all $n \geq N$. We are going to prove

$$
|g(x)-g(x+y)| \leq \varepsilon \quad \text { for every } y \in V_{N} .
$$

So $g$ will be continuous at $x$.

Take $n \geq N$ and $y \in V_{N}$. For every $z \in V_{n}$ we have $y+z \in W_{N}$ and $|f(x)-f(x+y+z)| \leq 1 / N$. By the definition of $f_{n}$ we get $\left|f(x)-f_{n}(x+y)\right|$ $\leq 1 / N$ for every $n \geq N$. Then we obtain (5) easily, since $f(x)=g(x)$.

REMARKS. (1) Actually the proof shows that if $\Lambda$ is a Lust-Piquard set and if there exists a surjective projection $Q: L_{\Lambda}^{\infty} \rightarrow \mathcal{C}_{\Lambda}$ which commutes with translations, then $\Lambda$ is a Rosenthal set.

(2) Talagrand's work [45] uses Martin's axiom, and in [46] another axiom is used, called $L$. But these axioms do not intervene in the results we use (they are needed to obtain Riemann-integrability from the weak measurability of translations: see [46, Theorem (15-4)]).

(3) F. Lust-Piquard and W. Schachermayer ([29, Corollary IV.4 and Proposition IV.15]; see also [11, Theorem V.1, Corollary VI.18, and Example VIII.10]) showed that if $L^{1}(G) / L_{\Gamma \backslash(-\Lambda)}^{1}$ does not contain $\ell_{1}$ (which is equivalent to $L_{\Lambda}^{\infty}$ having the weak Radon-Nikodym property [46, Corollary (7-3-8)]), then $L_{\Lambda}^{\infty}=R I_{\Lambda}$. Hence $\Lambda$ must be a Rosenthal set if $L_{\Lambda}^{\infty}$ has the weak Radon-Nikodym property and there exists a projection from $L_{\Lambda}^{\infty}$ onto $\mathcal{C}_{\Lambda}$. However, a direct proof is available. For a more general result, see [11, Example following Proposition VII.6].

(4) The first part of the proof is the same as the one used by A. Pełczyński $([34$, Cor. $9.4(\mathrm{a})])$ to show that $A(\mathbb{D})=\mathcal{C}_{\mathbb{N}}$ is not complemented in $H^{\infty}=L_{\mathbb{N}}^{\infty}$. 
Question. When $\Lambda$ is not a Rosenthal set, or merely when $\mathcal{C}_{\Lambda}$ contains $c_{0}$, how big can $L_{\Lambda}^{\infty} / \mathcal{C}_{\Lambda}$ be?

\section{References}

[1] J. Bourgain, Quelques propriétés linéaires topologiques de l'espace des séries de Fourier uniformément convergentes, Séminaire d'Initiation à l'Analyse, G. Choquet, M. Rogalski and J. Saint-Raymond (eds.), Publ. Math. Univ. Pierre et Marie Curie (Paris VI) 59 (1982/83), exp. no. 14.

[2] -, An approach to pointwise ergodic theorems, in: Lecture Notes in Math. 1317, Springer, 1988, 204-223.

[3] J. Bourgain and V. Milman, Dichotomie du cotype pour les espaces invariants, C. R. Acad. Sci. Paris Sér. I Math. 300 (1985), 263-266.

[4] M. Bożejko and T. Pytlik, Some types of lacunary Fourier series, Colloq. Math. 25 (1972), 117-124.

[5] J. Diestel, Applications of weak compactness and bases to vector measures and vectorial integration, Rev. Roumaine Math. Pures Appl. 18 (1973), 211-224.

[6] - Sequences and Series in Banach Spaces, Grad. Texts in Math. 92, Springer, 1984.

[7] J. Diestel and J. J. Uhl, Jr., Vector Measures, Math. Surveys 15, Amer. Math. Soc., 1977.

[8] J. J. F. Fournier, Two UC-sets whose union is not a UC-set, Proc. Amer. Math. Soc. 84 (1982), 69-72.

[9] J. Fournier and L. Pigno, Analytic and arithmetic properties of thin sets, Pacific J. Math. 105 (1983), 115-141.

[10] H. Furstenberg, Recurrence in Ergodic Theory and Combinatorial Number Theory, M. B. Porter Lectures, Princeton Univ. Press, 1981.

[11] N. Ghoussoub, G. Godefroy, B. Maurey and W. Schachermayer, Some topological and geometrical structures in Banach spaces, Mem. Amer. Math. Soc. 70 (1987), no. 378 .

[12] G. Godefroy, Existence and uniqueness of isometric preduals: a survey, in: Contemp. Math. 85, Amer. Math. Soc., 1989, 131-193.

[13] S. Hartman, Some problems and remarks on relative multipliers, Colloq. Math. 54 (1987), 103-111.

[14] N. Hindman, Ultrafilters and combinatorial number theory, in: Lecture Notes in Math. 751, Springer, 1979, 119-184.

[15] G. W. Johnson and G. S. Woodward, On p-Sidon sets, Indiana Univ. Math. J. 24 (1974), 161-167.

[16] J.-P. Kahane, Some Random Series of Functions, 2nd ed., Cambridge Stud. Adv. Math. 5, Cambridge Univ. Press, 1985.

[17] S. Kwapień and A. Pełczyński, Absolutely summing operators and translation invariant spaces of functions on compact abelian groups, Math. Nachr. 94 (1980), 303-340.

[18] P. Lefèvre, On some properties of the class of stationary sets, Colloq. Math. 76 (1998), 1-18.

[19] P. Lefèvre, D. Li, H. Queffélec and L. Rodríguez-Piazza, Lacunary sets and function spaces with finite cotype, J. Funct. Anal. 188 (2002), 272-291. 
[20] P. Lefèvre and L. Rodríguez-Piazza, p-Rider sets are q-Sidon sets, Proc. Amer. Math. Soc. 131 (2003), 1829-1838.

[21] D. Li, A class of Riesz sets, ibid. 119 (1993), 889-892.

[22] - On Hilbert sets and $\mathcal{C}_{\Lambda}(G)$-spaces with no subspace isomorphic to $c_{0}$, Colloq. Math. 68 (1995), 67-77.

[23] - Complex unconditional metric approximation property for $\mathcal{C}_{\Lambda}(\mathbb{T})$-spaces, Studia Math. 121 (1996), 231-247.

[24] D. Li, H. Queffélec and L. Rodríguez-Piazza, Some new thin sets in Harmonic Analysis, J. Anal. Math. 86 (2002), 105-138.

[25] F. Lust-Piquard, Propriétés géométriques des sous-espaces invariants par translation de $L^{1}(G)$ et $C(G)$, Séminaire sur la géométrie des espaces de Banach 1977-1978, École Polytechnique, Palaiseau, 1978, exp. no. 26.

[26] —, Eléments ergodiques et totalement ergodiques dans $L^{\infty}(G)$, Studia Math. 69 (1981), 191-225.

[27] —, Bohr local properties of $\mathcal{C}_{\Lambda}(\mathbb{T})$, Colloq. Math. 58 (1989), 29-38.

[28] - Means on $C V_{p}(G)$-subspaces of $C V_{p}(G)$ with $R N P$ and Schur property, Ann. Inst. Fourier (Grenoble) 39 (1989), 969-1006.

[29] F. Lust-Piquard and W. Schachermayer, Functions in $L^{\infty}(G)$ and associated convolution operators, Studia Math. 93 (1989), 109-136.

[30] Y. Meyer, Spectres des mesures et mesures absolument continues, ibid. 30 (1968), 87-99.

[31] I. M. Miheev, Trigonometric series with gaps, Anal. Math. 9 (1983), 43-55.

[32] M. B. Nathanson, Sumsets contained in infinite sets of integers, J. Combin. Theory Ser. A 28 (1980), 150-155.

[33] D. Oberlin, A Rudin-Carleson theorem for uniformly convergent Taylor series, Michigan Math. J. 27 (1980), 309-313.

[34] A. Pełczyński, Banach spaces of analytic functions and absolutely summing operators, CBMS Regional Conf. Ser. in Math. 30, Amer. Math. Soc., 1977.

[35] G. Pisier, Sur l'espace de Banach des séries de Fourier aléatoires presque sûrement continues, Séminaire sur la géométrie des espaces de Banach 1977-1978, École Polytechnique, Palaiseau, 1978, exp. no. 17-18.

[36] L. Rodríguez-Piazza, Caractérisation des ensembles p-Sidon p.s., C. R. Acad. Sci. Paris Sér. I Math. 305 (1987), 237-240.

[37] - Rango y propiedades de medidas vectoriales. Conjuntos p-Sidon p.s., thesis, Universidad de Sevilla, 1991.

[38] H. P. Rosenthal, On trigonometric series associated with weak ${ }^{*}$ closed subspaces of continuous functions, J. Math. Mech. 17 (1967), 485-490.

[39] L. A. Rubel and A. L. Shields, Invariant subspaces of $L^{\infty}$ and $H^{\infty}$, J. Reine Angew. Math. 272 (1975), 32-44.

[40] W. Rudin, Invariant means on $L^{\infty}$, Studia Math. 44 (1972), 219-227.

[41] S. Saccone, The Petczyński property for tight subspaces, J. Funct. Anal. 148 (1997), 86-116.

[42] - Function theory in spaces of uniformly convergent Fourier series, Proc. Amer. Math. Soc. 128 (2000), 1813-1823.

[43] P. M. Soardi and G. Travaglini, On sets of completely uniform convergence, Colloq. Math. 45 (1981), 317-320.

[44] M. Talagrand, Some functions with a unique invariant mean, Proc. Amer. Math. Soc. 82 (1981), 253-256.

[45] -, Closed convex hull of set of measurable functions, Riemann-measurable functions and measurability of translations, Ann. Inst. Fourier (Grenoble) 32 (1982), 39-69. 
[46] M. Talagrand, Pettis integral and measure theory, Mem. Amer. Math. Soc. 51 (1984), no. 307.

[47] S. A. Vinogradov, Convergence almost everywhere of Fourier series of functions in $L^{2}$ and the behaviour of the coefficients of uniformly convergent Fourier series, Soviet Math. Dokl. 17 (1976), 1323-1327.

Laboratoire de Mathématiques de Lens

Faculté des Sciences Jean Perrin

Université d'Artois

Rue Jean Souvraz, S.P. 18

62307 Lens Cedex, France

E-mail: pascal.lefevre@euler.univ-artois.fr daniel.li@euler.univ-artois.fr

Departamento de Análisis Matemático

Facultad de Matematicas

Universidad de Sevilla

Apartado de Correos 1160

41080 Sevilla, Spain

E-mail: piazza@us.es
Laboratoire A.G.A.T. U.F.R. de Mathématiques Université des Sciences et Techniques de Lille 59655 Villeneuve d'Ascq Cedex, France E-mail: queff@agat.univ-lille1.fr

Received January 31, 2003

Revised version November 17, 2003 